TRANSACTIONS OF THE

AMERICAN MATHEMATICAL SOCIETY

Volume 355, Number 5 , Pages 1961-1985

S 0002-9947(02)03150-1

Article electronically published on November 14, 2002

\title{
POTENTIAL THEORY ON LIPSCHITZ DOMAINS IN RIEMANNIAN MANIFOLDS: THE CASE OF DINI METRIC TENSORS
}

\author{
MARIUS MITREA AND MICHAEL TAYLOR
}

\begin{abstract}
We study the applicability of the method of layer potentials in the treatment of boundary value problems for the Laplace-Beltrami operator on Lipschitz sub-domains of Riemannian manifolds, in the case when the metric tensor $g_{j k} d x_{j} \otimes d x_{k}$ has low regularity. Under the assumption that

$$
\left|g_{j k}(x)-g_{j k}(y)\right| \leq C \omega(|x-y|),
$$

where the modulus of continuity $\omega$ satisfies a Dini-type condition, we prove the well-posedness of the classical Dirichlet and Neumann problems with $L^{p}$ boundary data, for sharp ranges of $p$ 's and with optimal nontangential maximal function estimates.
\end{abstract}

\section{INTRODUCTION}

In our papers [MT1]- MT4] we have developed the method of layer potentials as a tool to treat boundary problems for the Laplace operator and related operators on Lipschitz domains in Riemannian manifolds, extending work done on Lipschitz domains in Euclidean space with the standard flat metric, initiated in [FJR], [Ve, and $[\mathrm{DK}$. One of our goals has been to treat metrics of low regularity. In [MT1] we worked with $C^{1}$ metric tensors, extending this study to Lipschitz metric tensors in [MT2] and further, to Hölder continuous metric tensors, in [MT4]. Our main goal here is to extend the work of [MT4] to the case where the metric tensor is continuous, with a modulus of continuity satisfying a Dini-type condition, which will be described more precisely below.

In more detail, we consider operators of the form

$$
L=\Delta-V
$$

where $\Delta$ is the Laplace-Beltrami operator on a compact Riemannian manifold $M$ and $V \in L^{\infty}(M)$. Let $\Omega \subset M$ be a connected open set whose boundary is locally a Lipschitz graph. We will assume that $V \geq 0$ on $M$ and $V>0$ on a set of positive measure in each connected component of $M \backslash \bar{\Omega}$. We assume $M$ can be covered by coordinates in which the metric tensor is expressed as $g=\sum g_{j k} d x_{j} \otimes d x_{k}$, with

Received by the editors April 24, 2002 and, in revised form, July 4, 2002.

2000 Mathematics Subject Classification. Primary 31C12, 35J25, 45 E05.

The first author was partially supported by NSF grants DMS-9870018 and DMS-0139801.

The second author was partially supported by NSF grant DMS-9877077. 
the coefficients $g_{j k}$ satisfying

$$
\sum_{j, k}\left|g_{j k}(x)-g_{j k}(y)\right| \leq C \omega(|x-y|) .
$$

Here $\omega(h)$ is a modulus of continuity; i.e., $\omega:[0, \infty) \rightarrow[0, \infty)$ is continuous, vanishes at zero, and is slowly increasing (so there exists $C \in(0, \infty)$ such that $\omega(2 h) \leq C \omega(h)$, for all $h \in[0,1])$. We establish our results under the following hypothesis on $\omega$ :

$$
\omega(h)=\omega_{1}(h)^{2},
$$

where $\omega_{1}$ is a modulus of continuity satisfying the Dini condition

$$
\int_{0}^{1} \frac{\omega_{1}(t)}{t} d t<\infty
$$

We use boundary integral equation methods to treat the Dirichlet problem

$$
L u=0 \text { in } \Omega,\left.\quad u\right|_{\partial \Omega}=f \in L^{p}(\partial \Omega),
$$

and the Neumann problem

$$
L u=0 \text { in } \Omega,\left.\quad \partial_{\nu} u\right|_{\partial \Omega}=g \in L^{p}(\partial \Omega) .
$$

All boundary traces are taken in the nontangential limit sense (cf., e.g., [JK], $\mathrm{Ve}$, [MT1]). We also study the regularity problem, where $f \in H^{1, p}(\partial \Omega)$ in (1.5) (hereafter, $H^{s, p}$ will denote the standard scale of $L^{p}$-based Sobolev spaces of smoothness $s$ ), as well as oblique derivative problems.

In outline, the method of layer potentials goes as follows. Under our hypotheses on $L$, we have, for $p \in(1, \infty)$, that

$$
L: H^{1, p}(M) \longrightarrow H^{-1, p}(M)
$$

is an isomorphism. Denote by $E$ the integral kernel of $L^{-1}$, so

$$
L^{-1} u(x)=\int_{M} E(x, y) u(y) d \operatorname{Vol}(y), \quad x \in M,
$$

where $d \mathrm{Vol}$ is the volume element on $M$ determined by its Riemannian metric. For a function $f$ on $\partial \Omega$, define the single layer potential

$$
\mathcal{S} f(x)=\int_{\partial \Omega} E(x, y) f(y) d \sigma(y), \quad x \notin \partial \Omega,
$$

where $d \sigma$ is the natural area element on $\partial \Omega$, and define the double layer potential by

$$
\mathcal{D} f(x)=\int_{\partial \Omega} \frac{\partial E}{\partial \nu_{y}}(x, y) f(y) d \sigma(y), \quad x \notin \partial \Omega,
$$

where $\nu \in T^{*} M$ is the outward unit conormal to $\Omega$. The following properties were established in [MT1] MT2] for Lipschitz metric tensors and in [MT4] for Hölder continuous metric tensors, extending previously known results (of [Ve] et al.) for the flat Euclidean case. A major part of our work here will be to establish such properties when the metric tensor satisfies the conditions (1.2)-(1.4). 
Set $\Omega_{+}=\Omega$ and $\Omega_{-}=M \backslash \bar{\Omega}$. Given $f \in L^{p}(\partial \Omega), 1<p<\infty$, we have, for a.e. $x \in \partial \Omega$,

$$
\left.\mathcal{S} f\right|_{\partial \Omega_{+}}(x)=\left.\mathcal{S} f\right|_{\partial \Omega_{-}}(x)=S f(x),
$$

and

$$
\left.\mathcal{D} f\right|_{\partial \Omega_{ \pm}}(x)=\left( \pm \frac{1}{2} I+K\right) f(x)
$$

for operators $S$ and $K$ described in more detail in $\S 3$ (cf. (3.11)). Also, for a.e. $x \in \partial \Omega$,

$$
\left.\partial_{\nu} \mathcal{S} f\right|_{\partial \Omega_{ \pm}}(x)=\left\langle\nu(x),\left.\operatorname{grad} \mathcal{S} f\right|_{\partial \Omega_{ \pm}}(x)\right\rangle=\left(\mp \frac{1}{2} I+K^{*}\right) f(x),
$$

where $\langle\cdot, \cdot\rangle$ stands here for the duality pairing between $T^{*} M$ and $T M$. We have operator estimates for

$$
K, K^{*}: L^{p}(\partial \Omega) \longrightarrow L^{p}(\partial \Omega), \quad 1<p<\infty,
$$

and

$$
S: L^{p}(\partial \Omega) \longrightarrow H^{1, p}(\partial \Omega), \quad 1<p<\infty,
$$

plus natural accompanying nontangential maximal function estimates, i.e.,

$$
\left\|u^{*}\right\|_{L^{p}(\partial \Omega)} \leq C_{p}\|f\|_{L^{p}(\partial \Omega)}, \quad 1<p<\infty,
$$

with $u=\mathcal{D} f$ or $u=\nabla \mathcal{S} f$, and $u^{*}$ the nontangential maximal function of $u$. To define the latter, recall that if $\{\gamma(x)\}_{x \in \partial \Omega}$ is a family of nontangential approach regions (cf., e.g., [V], [MT1] for more details), then $u^{*}(x):=\sup \{|u(y)|: y \in \gamma(x)\}$ at each boundary point.

Next, invertibility results are demonstrated. Our main results to that effect, extending work in [FJR], $\mathrm{Ve},[\mathrm{DK}], \overline{\mathrm{KP}},[\mathrm{MT} 1-\mathrm{MT} 4$, are as follows. There is $\varepsilon=\varepsilon(\Omega)>0$ such that for each $2-\varepsilon<p<\infty, 1 / p+1 / q=1$, the operators

$$
\frac{1}{2} I+K: L^{p}(\partial \Omega) \longrightarrow L^{p}(\partial \Omega), \quad S: L^{q}(\partial \Omega) \longrightarrow H^{1, q}(\partial \Omega)
$$

are invertible. Also, if $V>0$ on a set of positive measure in $\Omega$, then

$$
-\frac{1}{2} I+K: L^{p}(\partial \Omega) \longrightarrow L^{p}(\partial \Omega)
$$

is invertible, while if $V=0$ on $\Omega$, then

$$
-\frac{1}{2} I+K^{*}: L_{0}^{q}(\partial \Omega) \longrightarrow L_{0}^{q}(\partial \Omega)
$$

is an isomorphism, where $L_{0}^{q}(\partial \Omega)$ consists of elements of $L^{q}(\partial \Omega)$ that integrate to zero. As a consequence, for $2-\varepsilon<p<\infty$, the Dirichlet problem (1.5) has a unique solution, which can be represented in the form

$$
u=\mathcal{D}\left(\left(\frac{1}{2} I+K\right)^{-1} f\right),
$$

and, for $1<p<2+\varepsilon$, the unique solution to the Neumann problem (1.6) is given by

$$
u=\mathcal{S}\left(\left(-\frac{1}{2} I+K^{*}\right)^{-1} g\right) .
$$

This analysis involves atomic Hardy space estimates and interpolation.

In the layer potential analyses of [FJR] and $\overline{\mathrm{Ve}}$, the operator estimates of $\mathrm{Ca}$ ] and [CMM] played a crucial role, and these estimates, and extensions established in [MT1, played a correspondingly crucial role in [MT1-MT2]. In the work in this paper to establish the results described above, when the metric tensor merely 
satisfies (1.2)-(1.4), the first major difficulty is to produce an analysis of the fundamental solution $E(x, y)$ sufficiently precise that these estimates from [MT1] are applicable. This central task is taken up in $\S 2$ of this paper, and indeed occupies much of its bulk. Once this analysis is carried out, we derive operator bounds, Fredholm properties, and invertibility results on operators of the form (1.14)-(1.19) in $\S 3$. We apply these results to the Dirichlet, Neumann, and regularity problems, for $p$ near 2 , in $\S 4$. In $\S 5$ we produce the atomic Hardy space estimates needed to extend these results to the optimal range of $p$. These arguments proceed along the lines of MT4, although further technical difficulties need to be overcome, particularly in a barrier function construction in $\S 5$.

We will use various classes of pseudodifferential operators, particularly in $\S 2$. We consider operators of the form

$$
\begin{aligned}
p(x, D) u & =(2 \pi)^{-n / 2} \int p(x, \xi) \hat{u}(\xi) e^{i x \cdot \xi} d \xi \\
& =(2 \pi)^{-n} \iint p(x, \xi) e^{i(x-y) \cdot \xi} u(y) d y d \xi
\end{aligned}
$$

Here $p(x, \xi)$ is the symbol of the operator $p(x, D)$, in a symbol class such as $S_{1, \delta}^{m}$, defined by

$$
p(x, \xi) \in S_{1, \delta}^{m} \Longleftrightarrow\left|D_{x}^{\beta} D_{\xi}^{\alpha} p(x, \xi)\right| \leq C_{\alpha \beta}\langle\xi\rangle^{m-|\alpha|+\delta|\beta|},
$$

where $\langle\xi\rangle=\left(1+|\xi|^{2}\right)^{1 / 2}$. Another class is $S_{\mathrm{cl}}^{m}$, consisting of $p(x, \xi) \in S_{1,0}^{m}$ with asymptotic expansions

$$
p(x, \xi) \sim \sum_{k \geq 0} p_{m-k}(x, \xi)
$$

where, for large $|\xi|, p_{m-k}(x, \xi)$ is homogeneous of degree $m-k$ in $\xi$. There are also various spaces of symbols with limited regularity, such as $C^{\omega} S_{1,0}^{m}$ and $C^{\omega} S_{\mathrm{cl}}^{m}$, defined as in [T1], [T3], and [MT1]- MT4]. If $p(x, \xi)$ belongs to a symbol space $X$, we say $p(x, D) \in O P X$. A variant of $(1.22)$ is

$$
q(D, x) u=(2 \pi)^{-n} \iint q(\xi, y) e^{i(x-y) \cdot \xi} u(y) d y d \xi
$$

If $q(\xi, x)$ belongs to a symbol space $X$, we say $q(D, x)$ belongs to $O P X$.

\section{Estimates on fundamental solutions for Dini Metric tensors}

Let $M$ be a compact, connected, smooth, $n$-dimensional manifold, with a Riemannian metric tensor, which we assume (unless otherwise stated) to be of class $C^{\omega}$, for some modulus of continuity $\omega$.

This metric gives rise to a Laplace operator,

$$
\Delta: H^{1, p}(M) \longrightarrow H^{-1, p}(M), \quad 1<p<\infty,
$$

defined by

$$
(\Delta u, v)=-\int_{M}\langle d u, d v\rangle d \mathrm{Vol}
$$

where the metric tensor determines the pointwise inner product $\langle\cdot, \cdot\rangle_{x}$ on $T_{x}^{*} M$ and the volume element $d$ Vol. In local coordinates (using the summation convention), 
provided the metric tensor has sufficient smoothness, we have

$$
\Delta u=\operatorname{div} \operatorname{grad} u=g^{-1 / 2} \partial_{j}\left(g^{j k} g^{1 / 2} \partial_{k} u\right),
$$

where $\left(g^{j k}\right)$ is the inverse matrix to $\left(g_{j k}\right)$, and $g=\operatorname{det}\left(g_{j k}\right)$. (Here, as well as on other subsequent occasions, the summation convention is used.) In fact, this makes sense for a Lipschitz metric tensor and $u \in H^{1, p}(M)$, but we aim to study cases where the metric tensor is less regular. Hence it is useful to keep in mind that $f \mapsto g^{1 / 2} f$ defines the correspondence between a locally integrable function on $M$ and a distribution. So if $f \in H^{-1, p}(M)$ is treated as a distribution, then the equation $\Delta u=f$ becomes, in local coordinates,

$$
\partial_{j}\left(g^{j k} g^{1 / 2} \partial_{k} u\right)=f .
$$

We will examine a fundamental solution on $M \times M$ of an operator of the form

$$
L=\Delta-V \text {. }
$$

We assume that $V \in L^{\infty}(M)$, that $V \geq 0$ on $M$, and that $V>0$ on a set of positive measure in $M$.

It is clear that, under these hypotheses on $V$ and whenever the metric tensor has components in $L^{\infty}$, the operator

$$
L: H^{1, p}(M) \rightarrow H^{-1, p}(M)
$$

is bounded for each $1<p<\infty$. Moreover, since $L$ is an isomorphism when $p=2$, it follows that there exists $\varepsilon>0$ such that $L$ in (2.6) is an isomorphism if $2-\varepsilon<p<2+\varepsilon$. Indeed, in the class of bounded metric tensors this invertibility range is optimal. This can be seen by means of a counterexample from [Me], a theme on which we briefly elaborate below.

Consider the two-dimensional torus $M:=\mathbb{T}^{2}=[-1,1] \times[-1,1] / \sim$, equipped with the $L^{\infty}$ Riemannian metric tensor $g$ which we now describe. Near the origin we take

$$
\begin{aligned}
& g_{11}(x, y)=1-\left(1-\mu^{2}\right) y^{2}\left(x^{2}+y^{2}\right)^{-1}, \\
& g_{12}(x, y)=g_{21}(x, y)=\left(1-\mu^{2}\right) x y\left(x^{2}+y^{2}\right)^{-1}, \\
& g_{22}(x, y)=1-\left(1-\mu^{2}\right) x^{2}\left(x^{2}+y^{2}\right)^{-1},
\end{aligned}
$$

where $\mu \in(0,1)$ is a fixed parameter, and then extend $g$ to the whole of $M$ so that $g_{j k} \in C^{\infty}(M \backslash\{0\})$. Note that the $g_{j k}$ 's are discontinuous at the origin and $g:=\operatorname{det}\left(g_{j k}\right) \equiv \mu^{2}$ near the origin. Denote by $\Delta_{g}$ the associated Laplace-Beltrami operator (as in (2.3)) and introduce the function $\tilde{u}(x, y):=x\left(x^{2}+y^{2}\right)^{(\mu-1) / 2} \in L^{\infty}$. A straightforward calculation shows that $\Delta_{g} \tilde{u}=0$ near the origin. Also, fix $\phi \in$ $C^{\infty}(M)$ so that $\phi \equiv 1$ near the origin, $\phi \equiv 0$ outside $|x| \leq 1 / 2$, and set $u:=\phi \tilde{u}$ (extended by zero outside of its support in $M$ ). It follows that

$$
u \in H^{1,2}(M), \quad \Delta_{g} u \in C^{\infty}(M),|\nabla u(x, y)| \approx\left(x^{2}+y^{2}\right)^{(\mu-1) / 2} \text { near }(0,0) .
$$

Consequently,

$$
u \in H^{1, p}(M) \Longleftrightarrow p<\frac{2}{1-\mu} .
$$

In particular, if the potential $V \in C^{\infty}(M)$ vanishes near zero, then $L$ in (2.6) fails to be an isomorphism for $p \geq 2 /(1-\mu)$. The fact that $2 /(1-\mu) \searrow 2$ as $\mu \searrow 0$ 
shows that this operator cannot be, generally speaking, an isomorphism for each $p>2$. By duality ( $L$ is formally selfadjoint), the same type of conclusion holds for $p<2$. Thus, some regularity of the metric is necessary if one expects $L$ in (2.6) to be an isomorphism for all $1<p<\infty$.

Turning to positive results, we note the following useful proposition. Here and elsewhere, vmo stands for the (local version of the) space of functions of vanishing mean oscillations.

Proposition 2.1. Assume the metric tensor $g$ on $M$ satisfies

$$
g_{j k} \in L^{\infty} \cap \text { vmo. }
$$

Then, for $1<p<\infty$, the map $L$ in (2.6) is an isomorphism.

This result is established in [DiF] and also in Proposition 1.7, Chapter 3, of [T3]. Proposition 2.1 implies that $L$ has a two-sided inverse

$$
E: H^{-1, p}(M) \longrightarrow H^{1, p}(M) \text {. }
$$

To be pedantic, one might denote this operator by $E_{p}$. However, it is clear that, if $v \in H^{-1, p} \cap H^{-1, q}$, then $E_{p} v=E_{q} v$; so we can drop the subscripts. By the Schwartz kernel theorem, $E$ defines an element of $\mathcal{D}^{\prime}(M \times M)$, which we also denote by $E$. Note also that, for $y \in M$, the Dirac point mass $\delta_{y}$ has the property

$$
\delta_{y} \in H^{r-1, p}(M), \quad p<\frac{n}{n-1}, r=r(p)>0 .
$$

Also, the map $y \mapsto \delta_{y}$ is a continuous map from $M$ to $H^{r-1, p}(M)$. Hence

$$
E(\cdot, y) \in H^{1, p}(M), \quad \forall p<\frac{n}{n-1},
$$

and the map $y \mapsto E(\cdot, y)$ is a continuous map from $M$ to $H^{1, p}(M)$.

We now make some estimates on $E(x, y)$ away from the diagonal. For this, we will use the following local regularity result, which follows from Proposition 1.10, Chapter 3, of [T3].

Proposition 2.2. Under the hypotheses of Proposition 2.1, given $1<p<q<$ $\infty, \mathcal{O} \subset M$ open,

$$
u \in H_{l o c}^{1, p}(\mathcal{O}), L u=f \in H_{l o c}^{-1, q}(\mathcal{O}) \Longrightarrow u \in H_{l o c}^{1, q}(\mathcal{O}) .
$$

If in addition the metric tensor is of class $C^{\omega}$ with

$$
\int_{0}^{1} \frac{\omega(t)}{t} d t<\infty, \quad t^{-\beta} \omega(t) \searrow, \text { for some } \beta \in(0,1),
$$

then, for $1<p<\infty$,

$$
u \in H_{l o c}^{1, p}(\mathcal{O}), L u=f \in C_{l o c}^{-1, \omega}(\mathcal{O}) \Longrightarrow u \in C_{l o c}^{1, \sigma}(\mathcal{O}),
$$

where

$$
\sigma(h)=\int_{0}^{h} \frac{\omega(t)}{t} d t .
$$

The spaces $C^{ \pm 1, \omega}$ are defined as follows. For $k \in \mathbb{Z}^{+}$, we set

$$
\begin{aligned}
C^{k, \omega} & =\left\{f: P f \in C^{\omega}, \forall P \in \operatorname{Diff}^{k}\right\}, \\
C^{-k, \omega} & =\left\{P g: g \in C^{\omega}, P \in \operatorname{Diff}^{k}\right\},
\end{aligned}
$$


where Diff ${ }^{k}$ denotes the class of differential operators of order $\leq k$, with smooth coefficients.

Going further, since $\delta_{y}$ is zero on $M \backslash\{y\}$, Proposition 2.2 gives:

Corollary 2.3. Under the hypotheses of Proposition 2.2, including (2.15),

$$
E(\cdot, y) \in C_{l o c}^{1, \sigma}(M \backslash\{y\}) .
$$

Now, $E(x, y)$ is symmetric in $x$ and $y$. So $E(x, \cdot)$ has the same sort of regularity. In particular, for $E \in \mathcal{D}^{\prime}(M \times M)$ we have behavior off the diagonal a bit better than

$$
E \in C_{\mathrm{loc}}^{1}(M \times M \backslash \operatorname{diag}) \text {. }
$$

It remains to investigate $E$ on a small neighborhood of the diagonal; this is the central point of our paper. Hence, given $y_{0} \in M$, we want to investigate $E$ on $\mathcal{O} \times \mathcal{O}$, where $\mathcal{O}$ is a coordinate neighborhood of $y_{0}$. Our subsequent calculations will be done in some local coordinate chart. For simplicity, we assume that $\operatorname{dim} M=n \geq 3$ in the calculations that follow. The modification for $n=2$ just involves using a logarithm in (2.21), which then gets dragged into a few of the subsequent estimates.

With $L$ written out in a coordinate system like that in $(2.3)$, let $E_{0}(D, x)$ denote the operator in $O P C^{\omega} S_{\mathrm{cl}}^{-2}$ with symbol $E_{0}(\xi, y)=-\left(g^{j k} \xi_{j} \xi_{k}\right)^{-1}$. The Schwartz kernel of $E_{0}(D, x)$ has the form

$$
e_{0}(x-y, y)=C\left(\sum g_{j k}(y)\left(x_{j}-y_{j}\right)\left(x_{k}-y_{k}\right)\right)^{-(n-2) / 2} .
$$

We will continue to regard $L$ as mapping functions to distributions, and hence omit the left factor $g^{-1 / 2}$ in (2.3). We have

$$
\begin{aligned}
& L E_{0}(D, x) u(x) \\
& \quad=(2 \pi)^{-n} \iint\left[-G(x, \xi)+a(x, \xi)-g(x)^{1 / 2} V(x)\right] E_{0}(\xi, y) e^{i(x-y) \cdot \xi} u(y) d y d \xi,
\end{aligned}
$$

with

$$
\begin{aligned}
G(x, \xi) & =\xi \cdot G(x) \xi, \quad G(x)=g(x)^{1 / 2}\left(g^{j k}(x)\right)_{j k}, \\
a(x, \xi) & =A(x) \xi=i \partial_{j}\left(g^{j k} g^{1 / 2}\right) \xi_{k} .
\end{aligned}
$$

Hence, writing

$$
-G(x, \xi) E_{0}(\xi, y)=g(x)^{1 / 2}-\xi \cdot[G(x)-G(y)] \xi E_{0}(\xi, y),
$$

we have

$$
L E_{0}(D, x) u(x)=g(x)^{1 / 2} u(x)+\int R(x, y) u(y) d y,
$$

where

$$
\begin{aligned}
R(x, y)= & (2 \pi)^{-n} \int \xi \cdot[G(y)-G(x)] \xi E_{0}(\xi, y) e^{i(x-y) \cdot \xi} d \xi \\
& +(2 \pi)^{-n} \int a(x, \xi) E_{0}(\xi, y) e^{i(x-y) \cdot \xi} d \xi \\
& +(2 \pi)^{-n} \int g(x)^{1 / 2} V(x) E_{0}(\xi, y) e^{i(x-y) \cdot \xi} d \xi \\
= & R_{1}(x, y)+R_{2}(x, y)+R_{3}(x, y),
\end{aligned}
$$


and the last equality defines $R_{j}(x, y), j=1,2,3$.

We first produce an estimate for $R_{1}(x, y)$. Pick $\Psi \in C_{0}^{\infty}\left(\mathbb{R}^{n}\right)$ with $\Psi(\xi)=1$ for $|\xi| \leq 1$. We have

$$
\begin{aligned}
R_{1}(x, y) & =\lim _{\mu \rightarrow \infty} \int \xi \cdot[G(y)-G(x)] \xi \Psi(\xi / \mu) E_{0}(\xi, y) e^{i(x-y) \cdot \xi} d \xi \\
& =\lim _{\mu \rightarrow \infty}[G(y)-G(x)] F_{\mu}(x-y, y)
\end{aligned}
$$

where

$$
F_{\mu}(z, y)=\mu^{n} F(\mu z, y),
$$

and $F(z, y)$ satisfies

$$
|F(z, y)| \leq C(1+|z|)^{-n}
$$

Hence

$$
\begin{aligned}
\left|[G(x)-G(y)] F_{\mu}(x-y, y)\right| & \leq C \frac{\mu^{n}|G(x)-G(y)|}{(1+\mu|x-y|)^{n}} \\
& \leq C \frac{\omega(|x-y|)}{|x-y|^{n}}\left(\frac{\mu|x-y|}{1+\mu|x-y|}\right)^{n}
\end{aligned}
$$

So passing to the limit as $\mu \rightarrow \infty$ gives

$$
\left|R_{1}(x, y)\right| \leq C \frac{\omega(|x-y|)}{|x-y|^{n}} .
$$

We hence have

$$
\int_{0}^{1} \frac{\omega(t)}{t} d t<\infty \Longrightarrow R_{1}(\cdot, y) \in L^{1} .
$$

We now tackle the term $R_{2}(x, y)$, which has the form

$$
R_{2}(x, y)=A(x) \Phi(x, y),
$$

with $\Phi$ the integral kernel of an operator in $\varnothing \mathrm{P} C^{\omega} S_{\mathrm{cl}}^{-1}$ and $A(x)$ is given in (2.23). In other words, we are looking at

$$
\left(\partial_{j} h\right) \Phi(x, y)=\partial_{j}(h \Phi)-h\left(\partial_{j} \Phi\right),
$$

with $\partial_{j}=\partial / \partial x_{j}$ and $h=h(x)$ an element of $C^{\omega}$. Now we can replace $h(x)$ by $h(x)-h(y)$ without altering (2.34). Thus we have

$$
R_{2}(x, y)=\nabla_{x}(h \Phi)-h\left(\nabla_{x} \Phi\right),
$$

with $h=h(x)-h(y)$, and with the estimates

$$
|h \Phi| \leq C \frac{\omega(|x-y|)}{|x-y|^{n-1}}, \quad\left|h \nabla_{x} \Phi\right| \leq C \frac{\omega(|x-y|)}{|x-y|^{n}} .
$$

Next we look at $R_{3}(x, y)$, which, by (2.26), obeys the estimate

$$
\left|R_{3}(x, y)\right| \leq C|x-y|^{-(n-2)},
$$

assuming $V \in L^{\infty}$.

We desire to estimate the difference

$$
e_{1}(x, y)=E(x, y) \sqrt{g(y)}-e_{0}(x-y, y)
$$


near the diagonal $x=y$. We are using the convention that, if a function $u$ is supported on a coordinate patch, then

$$
E u(x)=\int E(x, y) u(y) \sqrt{g(y)} d y .
$$

Note that $(2.25)$ gives

$$
L_{x} e_{0}(x-y, y)=\delta_{y}(x)+R(x, y)
$$

So we have

$$
L_{x} e_{1}(x, y)=-R(x, y) .
$$

Making use of the estimates on $R_{j}(x, y)$ established above, we aim to establish the following.

Proposition 2.4. Assume the metric tensor is of class $C^{\omega}$ and $\omega$ satisfies the Dini condition. Assume $\omega(h) / h^{\alpha} \searrow$ for some $\alpha \in(0,1)$. Then

$$
\left|e_{1}(x, y)\right| \leq C \frac{\sigma(|x-y|)}{|x-y|^{n-2}}
$$

with $\sigma(h)$ as in (2.17), and

$$
\left|\nabla_{x} e_{1}(x, y)\right| \leq C \frac{\beta(|x-y|)}{|x-y|^{n-1}},
$$

provided $\beta(h)$ satisfies the conditions

$$
\beta(h) \geq \sigma(h)=\int_{0}^{h} \frac{\omega(t)}{t} d t, \quad \omega(\rho h) \leq \beta(\rho) \omega_{1}(h),
$$

for $\rho, h \in(0,1]$, with $\omega_{1}(h)$ satisfying the Dini condition.

Proof. We can write $e_{1}(x, y)=e_{11}(x, y)+e_{12}(x, y)$, where

$$
L_{x} e_{11}(x, y)=\widetilde{R}_{1}(x, y), \quad\left|\widetilde{R}_{1}(x, y)\right| \leq C \frac{\omega(|x-y|)}{|x-y|^{n}},
$$

and

$$
L_{x} e_{12}(x, y)=\nabla_{x} S_{2}(x, y), \quad\left|S_{2}(x, y)\right| \leq C \frac{\omega(|x-y|)}{|x-y|^{n-1}} .
$$

Thus, bringing in the integral kernel $E(x, y)$, we have (recalling that the right sides of (2.45) and (2.46) are treated as distributions)

$$
\begin{aligned}
& e_{11}(x, y)=\int E(x, z) \widetilde{R}_{1}(z, y) d z, \\
& e_{12}(x, y)=-\int \nabla_{z} E(x, z) S_{2}(z, y) d z .
\end{aligned}
$$

Next, we shall make use of two estimates on $E(x, y)$ from [GW]. Namely,

$$
|E(x, y)| \leq \frac{C}{|x-y|^{n-2}},
$$

by Theorem 1.1 of GW, and

$$
\left|\nabla_{x} E(x, y)\right| \leq \frac{C}{|x-y|^{n-1}},
$$


by Theorem 3.3 of $\left[\mathrm{GW}\right.$. Hence, in order to estimate $e_{11}(x, y)$ and $\nabla_{x} e_{11}(x, y)$, we turn to the task of estimating

$$
\int_{|y| \leq 1}|x-y|^{-s} \frac{\omega(|y|)}{|y|^{n}} d y=A_{s}(x)
$$

in the cases $s=n-2$ and $s=n-1$, respectively. More generally, consider (2.50) for $1<s<n$.

Let $r=|x|$. We have

$$
\begin{aligned}
\int_{|y| \leq r / 2}|x-y|^{-s} \frac{\omega(|y|)}{|y|^{n}} d y & \leq C r^{-s} \int_{|y| \leq r / 2} \frac{\omega(|y|)}{|y|^{n}} d y \\
& \leq C r^{-s} \int_{0}^{r / 2} \frac{\omega(t)}{t} d t .
\end{aligned}
$$

Next,

$$
\begin{aligned}
\int_{r / 2 \leq|y| \leq 2 r}|x-y|^{-s} \frac{\omega(|y|)}{|y|^{n}} d y & \leq C \frac{\omega(r)}{r^{n}} \int_{r / 2 \leq|y| \leq 2 r}|x-y|^{-s} d y \\
& \leq C \frac{\omega(r)}{r^{n}} \int_{|z| \leq 4 r}|z|^{-s} d z \\
& \leq C \omega(r) r^{-s} .
\end{aligned}
$$

Furthermore, for $j \geq 1$,

$$
\int_{2^{j} r \leq|y| \leq 2^{j+1} r}|x-y|^{-s} \frac{\omega(|y|)}{|y|^{n}} d y \leq C \frac{\omega\left(2^{j} s\right)}{\left(2^{j} r\right)^{s}},
$$

and

$$
\begin{aligned}
\sum_{j \geq 1,2^{j} r \leq 1} \frac{\omega\left(2^{j} r\right)}{\left(2^{j} r\right)^{s}} & \leq C \int_{r}^{1} \frac{\omega(t)}{t^{s}} \frac{d t}{t} \\
& \leq C \frac{\omega(r)}{r} \int_{r}^{1} t^{-s} d t \\
& \leq C \omega(r) r^{-s} .
\end{aligned}
$$

Putting together (2.51)-(2.54), we get for (2.50) the estimate

$$
A_{s}(x) \leq C \sigma(|x|)|x|^{-s}, \quad 1<s<n,
$$

with $\sigma(h)$ given by $(2.44)$, and this yields $(2.42)-(2.43)$, with $e_{1}(x, y)$ replaced by $e_{11}(x, y)$, except for $n=3, s=n-2=1$. In this case, dominate the first integral on the right side of (2.54) by

$$
C \frac{\omega(r)}{r^{\alpha}} \int_{r}^{1} t^{-2+\alpha} d t \leq C \omega(r) r^{-1} .
$$

Note that we have an estimate on $\nabla_{x} e_{11}(x, y)$ better than that stated in (2.43), in that $\beta(|x-y|)$ can be replaced by $\sigma(|x-y|)$. 
In order to estimate $e_{12}(x, y)$, we note that, by symmetry, $\nabla_{z} E(x, z)$ satisfies an estimate parallel to (2.49). Thus in place of (2.50) we look at

$$
\int_{|y| \leq 1}|x-y|^{-s} \frac{\omega(|y|)}{|y|^{n-1}} d y=B_{s}(x)
$$

with $s=n-1$, or more generally for $2 \leq s<n$. This time, with $r=|x|$, we have

$$
\begin{aligned}
\int_{|y| \leq r / 2}|x-y|^{-s} \frac{\omega(|y|)}{|y|^{n-1}} d y & \leq C r^{-s} \int_{0}^{r / 2} \omega(t) d t \\
& \leq C r^{1-s} \omega(r),
\end{aligned}
$$

in place of (2.51). Analogues of (2.52)-(2.54) then produce the estimate

$$
B_{s}(x) \leq C \omega(|x|)|x|^{-s+1},
$$

for $2<s<n$, and a calculation parallel to (2.56) extends this to $s=2$. This gives an estimate of the type (2.42) (in an improved form) for $e_{12}(x, y)$.

It remains to establish an estimate of the form (2.43) for $e_{12}(x, y)$. While the estimate $\left|\nabla_{x} \nabla_{y} E(x, y)\right| \leq C|x-y|^{-n}$ is also established in Theorem 3.3 of [GW], it does not seem to work in an argument parallel to those given above, and we seem to need a different approach. We now turn to that task.

Note that by (2.49) and the estimate established on $\nabla_{x} e_{11}(x, y)$, plus the obvious estimate on $\nabla_{x} e_{0}(x-y, y)$, we have $\left|\nabla_{x} e_{12}(x, y)\right| \leq C|x-y|^{-(n-1)}$ at this point, but of course that is not satisfactory. To get off the ground, we establish an estimate on $e_{12}(x, y)$ that at least scales right under dilations.

We do this using spaces of Morrey type. We say

$$
f \in M_{q}^{p, \omega} \Longleftrightarrow\left(r^{-n} \int_{B_{r}}|f(x)|^{q} d x\right)^{1 / q} \leq C r^{-n / p} \omega(r),
$$

for all balls of radius $r \in(0,1)$, and then $M_{q}^{p, \omega}(M)$ is defined in an obvious fashion when $M$ is a compact Riemannian manifold. These spaces have been studied in $[\mathrm{P}],[\mathrm{Sp}$, and [T3]. From (2.46) we have, for each $y$,

$$
S_{2}(\cdot, y) \in M_{q}^{n /(n-1), \omega}, \quad \forall q<\frac{n}{n-1} .
$$

The following result will imply

$$
\nabla_{x} e_{12}(\cdot, y) \in M_{q}^{n /(n-1), \omega}, \quad \forall q<\frac{n}{n-1} .
$$

Proposition 2.5. Under the hypotheses of Proposition 2.4, if $1<q<p<\infty$, then

$$
\nabla u \in M_{q}^{p}, \quad L u=\partial_{j} f_{j}, \quad f_{j} \in M_{q}^{p, \omega} \Longrightarrow \nabla u \in M_{q}^{p, \omega},
$$

provided $t^{-\beta} \omega(t) \searrow$ for some $\beta \in(0, n / p)$.

Proof. As in the proof of a number of regularity results in [MT1-MT4 and in Chapter 3 of [T3], we use symbol smoothing to decompose $L$ into two pieces:

$$
L=L^{\#}+\partial_{j} A_{j}^{b},
$$


with

$$
L^{\#} \in O P S_{1, \delta}^{2} \text { elliptic, }
$$

where $\delta$ is chosen in $(0,1)$, and

$$
A_{j}^{b} \in O P C^{[\omega]} S_{1,(\tau)}^{1-\psi} .
$$

This follows by Proposition 3.4 in Chapter 1 of T3], which refines symbol smoothing results given in [T1]. The meaning of $(2.66)$ is that

$$
\begin{aligned}
\left|D_{\xi}^{\alpha} A_{j}^{b}(x, \xi)\right| & \leq A_{\alpha}\langle\xi\rangle^{1-\psi(\xi)-|\alpha|}, \\
\left\|D_{\xi}^{\alpha} A_{j}^{b}(\cdot, \xi)\right\|_{C^{[\omega]}} & \leq C_{\alpha} \tau(\xi)\langle\xi\rangle^{1-\psi(\xi)-|\alpha|} .
\end{aligned}
$$

The space $C^{[\omega]}$ is defined in (1.61), Chapter 1, of [T3; for here it is enough to know that $C^{[\omega]}=C^{\omega}$ whenever $t^{-\beta} \omega(t) \searrow$ for some $\beta \in(0,1)$. The functions $\psi(\xi)$ and $\tau(\xi)$ are described by (3.62) in Chapter 1 of [T3]. We can take

$$
\langle\xi\rangle^{-\psi(\xi)}=B(\langle\xi\rangle) \omega\left(\langle\xi\rangle^{-\delta}\right)=\tau(\xi)^{-1},
$$

where $B(\lambda) \geq 1$ is any smooth, monotonic function that satisfies $B(2 \lambda) \leq C B(\lambda)$ and

$$
\sum_{k \geq 0} \frac{1}{B\left(2^{k}\right)^{2}}<\infty
$$

Whenever $\omega(h)$ satisfies the Dini condition, one can find $B(\lambda)$ such that (2.68) and (2.69) hold with $\langle\xi\rangle^{-\psi(\xi)} \rightarrow 0$ as $|\xi| \rightarrow \infty$. For example, in the case

$$
\omega(h)=\left(\log \frac{1}{h}\right)^{-s}
$$

(with $s>1 / 2$ ), we can take

$$
\langle\xi\rangle^{-\psi(\xi)}=(\log \langle\xi\rangle)^{-s+1 / 2+\varepsilon}, \quad \tau(\xi)=(\log \langle\xi\rangle)^{s-1 / 2-\varepsilon},
$$

for any (small) $\varepsilon>0$. In general the symbol classes appearing here are well-behaved in the sense that

$$
O P C^{[\omega]} S_{1,(\tau)}^{0}: L^{p} \longrightarrow L^{p}, \quad 1<p<\infty,
$$

if, e.g., $\sum_{k \geq 1}\left[\omega\left(2^{-k}\right) \tau\left(2^{k}\right)\right]^{2}<\infty$; cf. Proposition 2.7, Chapter 1, in [T3].

Using the same localization techniques as in the proof of Proposition 1.10 in Chapter 3 of [T3, we can assume $M=\mathbb{T}^{n}$ (but with a non-flat metric).

We have a parametrix $E^{\#} \in O P S_{1, \delta}^{-2}$ for $L^{\#}$, and using it together with mapping properties established in Proposition 16.4 in Chapter 1 of [T3], we obtain that the maps

$$
\begin{gathered}
L^{\#}: M_{q}^{p, 1}(M) \longrightarrow M_{q}^{p,-1}(M), \\
L^{\#}: M_{q}^{p, \omega, 1}(M) \longrightarrow M_{q}^{p, \omega,-1}(M)
\end{gathered}
$$

are Fredholm, where, as in [T2], we set

$$
M_{q}^{p, s}(M)=\Lambda^{-s} M_{q}^{p}(M)
$$

and

$$
M_{q}^{p, \omega, s}(M)=\Lambda^{-s} M_{q}^{p, \omega}(M) .
$$


Here, $\Lambda=\left(I-\Delta_{0}\right)^{1 / 2}$, with $I$ and $\Delta_{0}$ being, respectively, the identity and the flat Laplacian on $M=\mathbb{T}^{n}$.

As for the remainder in (2.64), write

$$
A_{j}^{b}=B_{j \ell}^{b} \Psi(D) \partial_{\ell}, \quad \bmod O P C^{\omega} S^{-\infty},
$$

with

$$
B_{j \ell}^{b} \in O P C^{\omega} S_{1,(\tau)}^{0}, \quad \Psi(\xi)=\langle\xi\rangle^{-\psi(\xi)} .
$$

It is readily verified that, when acting on $M_{q}^{p}$ and also when acting on $M_{q}^{p, \omega}$, the operator $\Psi(D)$ is a norm limit of finite-rank operators, hence compact. Thus we have that

$$
\begin{gathered}
L: M_{q}^{p, 1}(M) \longrightarrow M_{q}^{p,-1}(M), \\
L: M_{q}^{p, \omega, 1}(M) \longrightarrow M_{q}^{p, \omega,-1}(M)
\end{gathered}
$$

are Fredholm. Considering now the one-parameter family $L_{t}=t \Delta+(1-t) \Delta_{0}-$ $t V-(1-t) I$ for $0 \leq t \leq 1$, we get a continuous scale of Fredholm operators $L_{t}$, with $L_{1}=L$ and $L_{0}$ invertible on the spaces in (2.78); so $L$ in (2.78) has index zero. We can apply Proposition 2.2 to see that elements in the kernel of $L$ all belong to $H^{1,2}(M)$, and hence vanish; so the operators in (2.78) are all invertible. The conclusion (2.63) is then immediate.

We are now in a position to complete the proof of Proposition 2.4, by establishing the following.

Lemma 2.6. In the setting of Proposition 2.4, we have

$$
\left|\nabla_{x} e_{12}(x, y)\right| \leq C \frac{\beta(|x-y|)}{|x-y|^{n-1}}
$$

provided

$$
\omega(\rho h) \leq \beta(\rho) \omega_{1}(h)
$$

for $\rho, h \in(0,1]$, with $\omega_{1}$ satisfying the Dini condition.

Proof. We suppose $x$ and $y$ belong to a coordinate neighborhood in $M$. Suppose $\left|x_{0}-y\right|=2 \rho$. We want to estimate $e_{12}(x, y)$ on $\left\{x:\left|x-x_{0}\right| \leq \rho / 2\right\}$. Shift coordinates so that $x_{0}=0$, and introduce the dilation operators

$$
u_{\rho}(x)=D_{\rho} u(x)=u(\rho x), \quad|x| \leq 1 .
$$

If $u(x)=e_{12}(x, y)$ for $|x|<\rho$, then $u_{\rho}$ satisfies the equation

$$
\widetilde{\Delta}_{\rho} u_{\rho}-\rho^{2} g_{\rho}^{1 / 2} V_{\rho} u_{\rho}=-\rho \nabla_{x} S_{2 \rho},
$$

where

$$
\widetilde{\Delta}_{\rho} v=\partial_{j} g_{\rho}^{j k} g_{\rho}^{1 / 2} \partial_{k} v
$$

and

$$
V_{\rho}(x)=V(\rho x), \quad S_{2 \rho}(x)=S_{2}(\rho x, \rho y), \quad g_{\rho}^{j k}(x)=g^{j k}(\rho x) .
$$

The equation (2.82) holds on $B_{1 / 2}$, where $B_{r}=\{x:|x| \leq r\}$. On this set, the collection of coefficients $g_{\rho}^{j k}$, etc., are of class $C^{\omega}$ and $\widetilde{\Delta}_{\rho}$ is elliptic, uniformly in $\rho \in\left(0, \rho_{0}\right]$ for some $\rho_{0}>0$. Recall that $S_{2}=h \Phi$ in $(2.35)$. Hence we have

$$
\left\|S_{2 \rho}\right\|_{L^{\infty}\left(B_{1 / 2}\right)} \leq C \rho^{-(n-1)} \omega(\rho) .
$$


Furthermore we see that, for $x, z \in B_{\rho / 2}\left(x_{0}\right)$,

$$
\begin{aligned}
\left|S_{2}(x)-S_{2}(z)\right| & \leq C \omega(|x-z|) \rho^{-(n-1)}+C \omega(\rho)|x-z| \rho^{-n} \\
& \leq C \omega(|x-z|) \rho^{-(n-1)},
\end{aligned}
$$

as long as $\omega(\rho) / \rho \searrow$. Consequently,

$$
\begin{aligned}
\left|S_{2 \rho}(x)-S_{2 \rho}(z)\right| & \leq C \omega(\rho|x-z|) \rho^{-(n-1)} \\
& \leq C \beta(\rho) \omega_{1}(|x-z|) \rho^{-(n-1)},
\end{aligned}
$$

for $x, z \in B_{1 / 2}$, and hence (2.85) is elevated to

$$
\left\|S_{2 \rho}\right\|_{C^{\omega_{1}\left(B_{1 / 2}\right)}} \leq C \rho^{-(n-1)} \beta(\rho) .
$$

Now the estimate $\left|e_{12}(x, y)\right| \leq C|x-y|^{-(n-2)} \omega(|x-y|)$ gives

$$
\left\|u_{\rho}\right\|_{L^{\infty}\left(B_{1 / 2}\right)} \leq C \rho^{-(n-2)} \omega(\rho),
$$

while the result $(2.62)$ gives, for any $q \in(1, n /(n-1))$,

$$
\left\|\nabla u_{\rho}\right\|_{L^{q}\left(B_{1 / 2}\right)} \leq C \rho^{-(n-2)} \omega(\rho) .
$$

We now apply the regularity result (2.16), with $\omega$ replaced by $\omega_{1}$ and $\sigma_{1}$ defined in the obvious fashion. There is a naturally accompanying estimate, giving

$$
\begin{aligned}
\left\|\nabla u_{\rho}\right\|_{C^{\sigma_{1}\left(B_{1 / 4}\right)} \leq} & C \rho\left\|S_{2 \rho}\right\|_{C^{\omega_{1}\left(B_{1 / 2}\right)}}+C\left\|\nabla u_{\rho}\right\|_{L^{q}\left(B_{1 / 2}\right)} \\
& +C\left\|u_{\rho}\right\|_{L^{\infty}\left(B_{1 / 2}\right)} \\
\leq & C \rho^{-(n-2)} \beta(\rho) .
\end{aligned}
$$

This is more than adequate to give (2.79). We have completed the proof of Lemma 2.6 and hence of Proposition 2.4.

In order for Proposition 2.4 to be useful for the study of layer potentials, we want $\sigma(h)$ and $\beta(h)$ to satisfy the Dini condition. This is guaranteed by the following.

Proposition 2.7. Suppose $\omega_{1}(h)$ satisfies the Dini condition and $\omega(h)=\omega_{1}(h)^{2}$. Then $\sigma(h)$ and $\beta(h)$ in (2.42)-(2.43) satisfy the Dini condition.

Proof. We have $\sigma(h) \leq \omega_{1}(h) \sigma_{1}(h)$ and $\omega(\rho h) \leq \omega_{1}(\rho) \omega_{1}(h)$; so we can take $\beta(h)=$ $\omega_{1}(h)$.

Clearly if $\omega(h)=h^{r}, 0<r<1$, we can do better, taking $\sigma(h) \approx \beta(h)=h^{r}$, but when $\omega(h)=(\log 1 / h)^{-s}$, one needs $s>2$, and the description above of $\sigma(h)$ and $\beta(h)$ is closer to optimal. More precisely, if $0<a<b$, we can take

$$
\begin{gathered}
\omega(h)=\left(\log \frac{1}{h}\right)^{-2-b}, \quad \sigma(h)=\left(\log \frac{1}{h}\right)^{-1-b}, \\
\beta(h)=\left(\log \frac{1}{h}\right)^{-1-a}, \quad \omega_{1}(h)=\left(\log \frac{1}{h}\right)^{-1-(b-a)} .
\end{gathered}
$$




\section{LAYER POTENTIALs For Dini METRIC TENSORS}

In the first half of this section we apply the results from $\S 2$ in order to derive estimates on layer potentials associated with a metric tensor of class $C^{\omega}$. Throughout the section, we retain the hypotheses made in $\S 1$ on $M$, the modulus of continuity $\omega$ and the potential $V$. Also, recall that $E=(\Delta-V)^{-1}$ is as in (2.11) and that $E(x, y)$ stands for the Schwartz kernel of $E$. Finally, we let $\nabla_{1} E(x, y)$ denote the $x$-gradient and $\nabla_{2} E(x, y)$ the $y$-gradient.

Theorem 3.1. Assume $M$ is a compact, connected, smooth manifold equipped with a metric tensor $g$ of class $C^{\omega}$, with $\omega$ as in (1.3)-(1.4). Fix $\bar{\Omega}$, a domain in $M$ with nonempty Lipschitz boundary, and denote by $\nu \in T^{*} M$ the (outward) unit conormal and by $d \sigma$ the surface measure on $\partial \Omega$ (both determined by the Riemannian metric on $M)$.

Next, introduce the (vector-valued) integral operators

$$
\mathcal{T}_{j} f(x):=\int_{\partial \Omega} \nabla_{j} E(x, y) f(y) d \sigma(y), \quad x \notin \partial \Omega,
$$

and

$$
T_{j} f(x):=p \cdot v \cdot \int_{\partial \Omega} \nabla_{j} E(x, y) f(y) d \sigma(y), \quad x \in \partial \Omega,
$$

for $j=1,2$, where $f$ is a scalar-valued function on $\partial \Omega$. Here p.v. indicates that the integral is taken in the principal value sense, i.e., by removing small geodesic balls with respect to some smooth background metric and then passing to the limit.

Then, for $1<p<\infty$,

$$
\begin{aligned}
\left\|\left(\mathcal{T}_{j} f\right)^{*}\right\|_{L^{p}(\partial \Omega)} & \leq C\|f\|_{L^{p}(\partial \Omega)}, & j=1,2, \\
\left\|T_{j} f\right\|_{L^{p}(\partial \Omega)} & \leq C\|f\|_{L^{p}(\partial \Omega)}, & j=1,2,
\end{aligned}
$$

and, for each $f \in L^{p}(\partial \Omega), 1<p<\infty$, the following jump-relations hold:

$$
\left.\mathcal{T}_{j} f\right|_{\partial \Omega_{ \pm}}= \pm(-1)^{j} \frac{1}{2} f \nu+T_{j} f
$$

a.e. on $\partial \Omega$.

Here and elsewhere, $\Omega_{+}:=\Omega$ and $\Omega_{-}:=M \backslash \bar{\Omega}$.

Proof. Recall from $\S 2$ that

$$
E(x, y)=g(y)^{-1 / 2} e_{0}(x-y, y)+g(y)^{-1 / 2} e_{1}(x, y),
$$

where $e_{0}(x-y, y)$ has been introduced in (2.21) and $e_{1}(x, y)$ satisfies the estimates in Proposition 2.4.

Consider the case $j=1$ in (3.3)-(3.4). The contribution from the first term in (3.5) can be handled as in Propositions 1.4-1.6 of [MT1. Also, granted the estimates (2.42)-(2.43) on $e_{1}(x, y)$, the contribution from the second term in (3.5) is amenable to an elementary treatment, which we omit.

For the case $j=2$ in (3.3)-(3.4), the symmetry condition

$$
E(x, y)=E(y, x)
$$

in concert with (3.5) gives

$$
E(x, y)=g(x)^{-1 / 2} e_{0}(x-y, x)+g(x)^{-1 / 2} e_{1}(y, x) .
$$

In this form, the previous pattern of reasoning applies once again. The theorem is hence proved. 
With the same assumptions as in Theorem 3.1, recall from $\S 1$ the single and double layer potentials associated with $\Omega$.

Corollary 3.2. In the setting of Theorem 3.1, for $1<p<\infty$ we have

$$
\left\|(\nabla \mathcal{S} f)^{*}\right\|_{L^{p}(\partial \Omega)} \leq C\|f\|_{L^{p}(\partial \Omega)}, \quad\left\|(\mathcal{D} f)^{*}\right\|_{L^{p}(\partial \Omega)} \leq C\|f\|_{L^{p}(\partial \Omega)},
$$

uniformly for $f \in L^{p}(\partial \Omega)$.

Also, given $1<p<\infty$ and $f \in L^{p}(\partial \Omega)$, then for a.e. $x \in \partial \Omega$ we have

$$
\left.\mathcal{S} f\right|_{\partial \Omega_{+}}(x)=\left.\mathcal{S} f\right|_{\partial \Omega_{-}}(x)=S f(x)
$$

and

$$
\left.\mathcal{D} f\right|_{\partial \Omega_{ \pm}}(x)=\left( \pm \frac{1}{2} I+K\right) f(x)
$$

where, for $x \in \partial \Omega$,

$$
S f(x):=\int_{\partial \Omega} E(x, y) f(y) d \sigma(y), \quad K f(x):=p . v . \int_{\partial \Omega} \frac{\partial E}{\partial \nu_{y}}(x, y) f(y) d \sigma(y) .
$$

The principal value integral above is understood in the sense of removing small geodesic balls (with respect to some smooth background metric) and then passing to the limit.

Furthermore,

$$
\left.\partial_{\nu} \mathcal{S} f\right|_{\partial \Omega_{ \pm}}(x)=\left(\mp \frac{1}{2} I+K^{*}\right) f(x) .
$$

Finally, the boundary integral operators

$$
\pm \frac{1}{2} I+K, \pm \frac{1}{2} I+K^{*}: L^{p}(\partial \Omega) \rightarrow L^{p}(\partial \Omega), \quad S: L^{p}(\partial \Omega) \rightarrow H^{1, p}(\partial \Omega)
$$

are well-defined and bounded for $1<p<\infty$.

In the second part of this section we tackle the issue of invertibility for the operators in (3.13). The main idea is to implement an approximation procedure, along the lines of $\S 3$ in MT4. To this end, we take a sequence $g^{\mu}$ of $C^{1}$ metric tensors on $M$ so that

$$
\begin{aligned}
& \left\{g^{\mu}: \mu \in \mathbb{Z}^{+}\right\} \text {is bounded in } L^{\infty}, \\
& g^{\mu} \longrightarrow g \text { uniformly on } \partial \Omega \text {, as } \mu \rightarrow \infty .
\end{aligned}
$$

For each metric $g$, let $E_{g}(x, y)$ stand for the Schwartz kernel of $\left(\Delta_{g}-V\right)^{-1}$ and, generally speaking, let the subscript $g$ indicate that the corresponding object is constructed in connection with the metric $g$. Our main estimate is contained in the following.

Proposition 3.3. Let $\mathcal{K}\left(L^{p}(\partial \Omega)\right)$ denote the closed subspace of $\mathcal{L}\left(L^{p}(\partial \Omega)\right)$, the collection of all bounded operators on $L^{p}(\partial \Omega)$, consisting of compact operators. Then, for each $p \in(1, \infty)$, the norms

$$
\left\|K_{g}-K_{g^{\mu}}\right\|_{\mathcal{L}\left(L^{p}(\partial \Omega)\right) / \mathcal{K}\left(L^{p}(\partial \Omega)\right)}, \quad\left\|K_{g}^{*}-K_{g^{\mu}}^{*}\right\|_{\mathcal{L}\left(L^{p}(\partial \Omega)\right) / \mathcal{K}\left(L^{p}(\partial \Omega)\right)},
$$

and

$$
\left\|\nabla S_{g}-\nabla S_{g^{\mu}}\right\|_{\mathcal{L}\left(L^{p}(\partial \Omega)\right) / \mathcal{K}\left(L^{p}(\partial \Omega)\right)}
$$

can be made as small as we please by selecting $\mu$ sufficiently large. 
Proof. Consider, for example, the case of (3.16). Based on the decomposition (3.5), locally we can write

$$
\begin{aligned}
\nabla S_{g} f(x)= & \int_{\partial \Omega} \nabla_{x} e_{0, g}(x-y, y) g(y)^{-1 / 2} f(y) d \sigma_{g}(y) \\
& +\int_{\partial \Omega} \nabla_{x} e_{1, g}(x, y) g(y)^{-1 / 2} f(y) d \sigma_{g}(y) \\
= & : T_{0, g} f(x)+T_{1, g} f(x) .
\end{aligned}
$$

Now, the estimate

$$
\left\|T_{0, g}-T_{0, g^{\mu}}\right\|_{\mathcal{L}\left(L^{p}(\partial \Omega)\right)} \leq C\left\|g^{\mu}-g\right\|_{L^{\infty}(\partial \Omega)}
$$

has been established in MT4. So it only remains to show that $T_{1, g}$ is a compact operator on $L^{p}$. However, granted the estimates on $e_{1}(x, y)$ from $\S 2$, this is a simple consequence of the elementary lemma below. Modulo this, the proof of Proposition 3.3 is finished.

Lemma 3.4. Let

$$
T f(x):=\int_{|y| \leq 1} K(x, y) f(y) d y, \quad|x| \leq 1,
$$

be an integral operator whose kernel satisfies

$$
\begin{gathered}
K \in L_{\text {loc }}^{\infty}\left(B_{1} \times B_{1} \backslash \text { diag }\right) \text { and } \\
|K(x, y)| \leq k(x-y) \text { for some } k \in L^{1}\left(B_{2}\right) .
\end{gathered}
$$

Then, for each $p \in(1, \infty)$, the map $T: L^{p}\left(B_{1}\right) \rightarrow L^{p}\left(B_{1}\right)$ is compact.

Proof. For $j=1,2, \ldots$, set $K_{j}(x, y):=K(x, y) \chi_{\{|x-y| \geq 1 / j\}}$ and denote by $T_{j}$ the integral operator associated with $K_{j}$. Since $K_{j} \in L^{\infty}\left(B_{1} \times B_{1}\right)$, it follows that $T_{j}$ is compact on $L^{p}\left(B_{1}\right)$; see, e.g., $§ 9.5$ in [Ed]. On the other hand, $\left|K(x, y)-K_{j}(x, y)\right| \leq$ $k(x-y) \chi_{\{|x-y|<1 / j\}}$, so that $\left\|T-T_{j}\right\|_{\mathcal{L}\left(L^{p}\left(B_{1}\right)\right)} \rightarrow 0$ as $j \rightarrow \infty$, by Schur's lemma, for each $1<p<\infty$. The desired conclusion follows.

We are now in a position to discuss the following invertibility results for layer potentials.

Theorem 3.5. Under the same assumptions as in Theorem 3.1, there exists $\varepsilon=$ $\varepsilon(\Omega, L)>0$ such that for $2-\varepsilon<p<2+\varepsilon$, the maps

$$
\frac{1}{2} I+K, \frac{1}{2} I+K^{*}: L^{p}(\partial \Omega) \longrightarrow L^{p}(\partial \Omega)
$$

and

$$
S: L^{p}(\partial \Omega) \longrightarrow H^{1, p}(\partial \Omega)
$$

are isomorphisms.

Moreover, for the same range of $p$ 's, if $V>0$ on a set of positive measure in $\Omega$, then $-\frac{1}{2} I+K$ and $-\frac{1}{2}+K^{*}$ are invertible on $L^{p}(\partial \Omega)$, whereas if $V=0$ on $\bar{\Omega}$, then

$$
-\frac{1}{2} I+K^{*}: L_{0}^{p}(\partial \Omega) \longrightarrow L_{0}^{p}(\partial \Omega)
$$

is an isomorphism. Here $L_{0}^{p}(\partial \Omega)$ consists of $L^{p}$-functions integrating to zero on $\partial \Omega$. 
Proof. With the main ingredients in place, the proof proceeds as in [MT4. The departure point is to approximate the original metric $g$ as in (3.14) and to write Rellich estimates for each approximating metric $g^{\mu}$. These amount to the boundedness from below of the layer potentials under discussion, associated with $g^{\mu}$, modulo compacts. The crux of the matter is that, since the relevant constants are independent of $\mu$, an application of Proposition 3.3 allows us to conclude the same thing about the layer potentials associated with the original metric $g$. In turn, this entails the semi-Fredholmness of the operators in question. The rest is routine, and we refer the interested reader to [MT4] for more details in similar circumstances.

It is also of interest to complement the statement of Theorem 3.5 with certain uniform invertibility results. More specifically, consider a suitable approximation of $\Omega$ by smooth domains $\Omega_{j} \nearrow \Omega$ (cf. Ve], MT1). Among other things, it is assumed that the smooth boundaries $\partial \Omega_{j}$ have Lipschitz constants bounded uniformly in $j$. Below we shall use the subscript $j$ to label layer potential operators constructed in connection with $\partial \Omega_{j}$ rather than $\partial \Omega$.

Proposition 3.6. Retain the same hypotheses as in the previous theorem. Also, assume that $V>0$ on a set of positive measure in $\Omega$. There exists $\varepsilon>0$ and a constant $0<C_{0}<\infty$, independent of $j$, such that

$$
\begin{aligned}
& \|f\|_{L^{p}\left(\partial \Omega_{j}\right)} \leq C_{0}\left\|\left(-\frac{1}{2} I+K_{j}^{*}\right) f\right\|_{L^{p}\left(\partial \Omega_{j}\right)}, \quad \forall f \in L^{p}\left(\partial \Omega_{j}\right), \\
& \|f\|_{L^{p}\left(\partial \Omega_{j}\right)} \leq C_{0}\left\|\left(\frac{1}{2} I+K_{j}\right) f\right\|_{L^{p}\left(\partial \Omega_{j}\right)}, \quad \forall f \in L^{p}\left(\partial \Omega_{j}\right), \\
& \|f\|_{L^{p}\left(\partial \Omega_{j}\right)} \leq C_{0}\left\|S_{j} f\right\|_{H^{1, p}\left(\partial \Omega_{j}\right)}, \quad \forall f \in L^{p}\left(\partial \Omega_{j}\right),
\end{aligned}
$$

for $2-\varepsilon<p<2+\varepsilon$. In the case where $V=0$ on a neighborhood of $\bar{\Omega}$, then $L^{p}\left(\partial \Omega_{j}\right)$ should be replaced by $L_{0}^{p}\left(\partial \Omega_{j}\right)$ for the first estimate in (3.24).

Proof. This has been proved in $\S 3$ of [MT4] under the stronger assumption that the metric tensor $g$ is Hölder continuous. However, an inspection of the proof reveals that Proposition 3.3 suffices for the task at hand.

\section{Boundary VAlue PRoblems}

We continue to enforce the hypotheses on $M, \Omega$, and $L=\Delta-V$ made in $\S 1$. In particular, we assume that the metric tensor $g$ is of class $C^{\omega}$ with $\omega$ as in (1.3)-(1.4). Furthermore, $\sigma$ is given by (2.17). The aim of this section is to discuss the classical boundary problems, i.e., the Dirichlet (and its "regular" version), the Neumann (and its Robin version) and oblique derivative problems. Here is our first result.

Theorem 4.1. There exists $\varepsilon=\varepsilon(\Omega, L)>0$ such that the following is true for $2-\varepsilon<p<2+\varepsilon$. Given $f \in L^{p}(\partial \Omega)$, there exists a unique function $u \in C_{l o c}^{1, \sigma}(\Omega)$ satisfying

$$
L u=0 \text { on } \Omega, \quad u^{*} \in L^{p}(\partial \Omega),\left.\quad u\right|_{\partial \Omega}=f .
$$

Also, $\left\|u^{*}\right\|_{L^{p}(\partial \Omega)} \leq C\|f\|_{L^{p}(\partial \Omega)}$ and $u$ is representable in the form

$$
u=\mathcal{D}\left(\left(\frac{1}{2} I+K\right)^{-1} f\right) \quad \text { in } \Omega .
$$

Moreover, for the same range of $p$ 's,

$$
u \in C_{l o c}^{1}(\Omega) \text { and }(\nabla u)^{*} \in L^{p}(\partial \Omega) \Longleftrightarrow f \in H^{1, p}(\partial \Omega) .
$$


Natural norm estimates accompany this equivalence. In this latter case, $u$ can also be expressed as

$$
u=\mathcal{S}\left(S^{-1} f\right) \quad \text { in } \Omega .
$$

Proof. As Theorem 3.5 and Corollary 3.2 show, $u$ given by (4.2) is well-defined and solves (4.1). Uniqueness follows by considering $\Omega_{j} \nearrow \Omega$ and then constructing a sequence of Green functions, corresponding to each $\Omega_{j}$, with control of their gradients uniform in $j$. That this works in the present situation is guaranteed by Proposition 3.7. See Proposition 9.1 of [MT1] for more details in the case of $C^{1}$ metric tensors.

Finally, (4.3) and (4.4) are once again consequences of Theorem 3.5 and Corollary 3.2. The proof is complete.

Our next result deals with the Neumann and Robin problems.

Theorem 4.2. For each $\lambda \in L^{\infty}(\partial \Omega)$, there exists $\varepsilon=\varepsilon(\Omega, L, \lambda)>0$ with the following property. If $2-\varepsilon<p<2+\varepsilon$, then the Robin boundary value problem

$$
u \in C_{l o c}^{1, \sigma}(\Omega), \quad L u=0, \quad(\nabla u)^{*} \in L^{p}(\partial \Omega),\left.\quad \partial_{\nu} u\right|_{\partial \Omega}+\left.\lambda u\right|_{\partial \Omega}=f \in L^{p}(\partial \Omega)
$$

is solvable if and only if the boundary datum $f$ satisfies finitely many (necessary) linear conditions. Moreover, the solution is unique modulo a finite-dimensional linear space (whose dimension coincides with the number of linearly independent constraints required for the boundary data).

If, in addition, $\lambda \geq 0$ a.e. on $\partial \Omega$, we can be more specific. Concretely, there are two cases to discuss.

(i) If $V>0$ on a set of positive measure in $\Omega$ or if $V \equiv 0$ in $\Omega$ but $\lambda>0$ on a set of positive surface measure, then (4.5) is uniquely solvable for each $f \in L^{p}(\partial \Omega)$. In this situation, $u$ satisfies

$$
\left\|(\nabla u)^{*}\right\|_{L^{p}(\partial \Omega)} \leq C\|f\|_{L^{p}(\partial \Omega)}
$$

and is given by

$$
u=\mathcal{S}\left(\left(-\frac{1}{2} I+K^{*}+\lambda S\right)^{-1} f\right) \quad \text { in } \Omega .
$$

(ii) If both $V \equiv 0$ in $\Omega$ and $\lambda \equiv 0$ on $\partial \Omega$, then (4.5) is solvable if and only if $\int_{\partial \Omega} f d \sigma=0$. In this latter case, the solution is unique up to an additive constant and (4.6) continues to hold.

Proof. First, there is the issue of representability of any function $u$ satisfying the first three conditions in (4.5) in the form $u=\mathcal{S} h$, for some $h \in L^{p}(\partial \Omega)$. This depends on the well-posedness of the regular version of the Dirichlet problem (Theorem 4.1) plus a Fatou-type result for the gradients of null-solutions of the operator $L=\Delta-V$. The latter amounts to the fact that if $u \in C_{\mathrm{loc}}^{1}(\Omega)$ satisfies

$$
L u=0 \text { in } \Omega, \quad(\nabla u)^{*}(x)<+\infty \text { for a.e. } x \in \partial \Omega,
$$

then the pointwise nontangential boundary trace $\left.\nabla u\right|_{\partial \Omega}$ exists at almost every point of $\partial \Omega$. The proof is essentially the same as that of Proposition 3.1 in [MT2]. The argument used there also works in the present setting, thanks to Proposition 3.6 of this paper.

With this at hand, it follows that (4.5) is solvable if and only if the boundary datum $f$ belongs to Range $\left(T ; L^{p}(\partial \Omega)\right)$, where $T:=-\frac{1}{2} I+K^{*}+\lambda S$. Note that 
from the results in $\S 3$, the operator $T$ is Fredholm with index zero on $L^{p}(\partial \Omega)$ for each $\lambda \in L^{\infty}(\partial \Omega)$. Thus, with $\{\ldots\}^{\circ} \subset L^{p}$ denoting the annihilator of $\{\ldots\} \subseteq L^{q}$, $1 / p+1 / q=1$, the membership

$$
f \in \operatorname{Range}\left(T ; L^{p}(\partial \Omega)\right)=\left\{\operatorname{Ker}\left(T^{*} ; L^{q}(\partial \Omega)\right)\right\}^{\circ}
$$

translates into a set of finitely many (necessary) linear conditions which the boundary datum must satisfy.

Let us also note that the kernel of $T$ on $L^{p}(\partial \Omega)$ is in a one-to-one and onto correspondence with the space of null-solutions of the boundary problem (4.5). In particular, since $T$ has index zero, this shows that the space of null-solutions of (4.5) has the same (finite) dimension as the number of linear compatibility conditions which the boundary datum $f$ should satisfy. Clearly, this amounts to saying that the index of the problem (4.5) is zero.

Next we turn our attention to the situation when $\lambda \geq 0$ on $\partial \Omega$, and consider case $(i)$ in the statement of the theorem. The claim is that the operator $T$ above is actually invertible on $L^{p}(\partial \Omega)$ for $2-\varepsilon<p<2+\varepsilon$. Indeed, it suffices to show that this is the case when $p=2$. The case of other values of $p$ then follows from this and elementary functional analysis. (Indeed, $T$ is a Fredholm operator with index zero on $L^{p}(\partial \Omega)$, which has dense range for $p<2$ (since $L^{2}(\partial \Omega) \hookrightarrow L^{p}(\partial \Omega)$ densely in this case) and is one-to-one for $p>2$ (since, this time, $L^{p}(\partial \Omega) \hookrightarrow L^{2}(\partial \Omega)$ ). Thus, in either case, the operator in question is invertible.)

Returning to the analysis of the case $p=2$, if $h$ belongs to the kernel of $T$ on $L^{2}(\partial \Omega)$ and $u:=\mathcal{S} h$ in $\Omega$, we can use Green's formula to write

$$
\int_{\Omega}\left\{|\nabla u|^{2}+V|u|^{2}\right\} d \mathrm{Vol}=-\int_{\partial \Omega} \lambda|u|^{2} d \sigma .
$$

Now, the current assumptions on $V$ and $\lambda$ force $u \equiv 0$ in $\Omega$ and, further, by going to the boundary, $S h=0$ in $L^{2}(\partial \Omega)$. Thus $h=0$, by Theorem 3.3. This shows that $T$, on top of being Fredholm with index zero, is also one-to-one. The desired conclusion readily follows.

Finally, in case (ii), i.e., when $V \equiv 0$ and $\lambda \equiv 0$, essentially the same reasoning as above shows that the kernel of $T$ precisely consists of constants. This, of course, suffices to conclude the proof of the theorem.

Next we discuss the oblique derivative problem for $L$ under our standard assumptions on $M$, the metric, $\Omega$, and $L$. This extends the work in [MT1] and [MT4], where the case of $C^{1}$ and Hölder metrics has been treated. Further references to related work are also given in [MT1.

Theorem 4.3. Let $w \in L^{\infty}(\partial \Omega) \cap$ vmo $(\partial \Omega)$ be a transversal vector field to $\partial \Omega$, i.e., assume there exists a constant $C_{0}$ for which

$$
\langle\nu, w\rangle \geq C_{0}>0 \text { a.e. on } \partial \Omega \text {. }
$$

Also, fix some $\lambda \in L^{\infty}(\partial \Omega)$.

Then there exists an $\varepsilon>0$, small, depending only on $\partial \Omega, w$ and $\lambda$, such that for $2-\varepsilon<p<2+\varepsilon$ the following holds. For any $f \in L^{p}(\partial \Omega)$ satisfying finitely many (necessary) linear conditions, there exists $u \in C_{\text {loc }}^{1, \sigma}(\Omega)$ with

$$
L u=0, \quad(\nabla u)^{*} \in L^{p}(\partial \Omega), \quad \text { and }\left.\quad \partial_{w} u\right|_{\partial \Omega}+\left.\lambda u\right|_{\partial \Omega}=f .
$$


Also, the solution is unique modulo a finite-dimensional linear space (whose dimension coincides with the number of linearly independent constraints required for the boundary data). In particular, the index of the problem is zero.

Proof. Our solution closely parallels that of Theorem 11.1 in [MT1] and Theorem 8.1 in [MT4]. As such, there is only one aspect which, in the case of a metric tensor satisfying a Dini condition, must be reconsidered.

Specifically, the solution given in [MT1 and [MT4 is based on the observation that a certain integral kernel on $\partial \Omega$ gives rise to a compact operator on $L^{p}(\partial \Omega)$. The estimate on the kernel which has been established in [MT4] is

$$
\left|\nabla_{1} e_{0}(x-y, x)-\nabla_{1} e_{0}(x-y, y)\right| \leq C|x-y|^{-(n-1)} \sum_{j, k}\left|g_{j k}(x)-g_{j k}(y)\right| .
$$

Clearly, if the metric tensor has Hölder components, then this expression is just weakly singular on $\partial \Omega$, and the conclusion follows.

In our case, (4.13) only yields

$$
\left|\nabla_{1} e_{0}(x-y, x)-\nabla_{1} e_{0}(x-y, y)\right| \leq C \frac{\omega(|x-y|)}{|x-y|^{n-1}},
$$

which, nonetheless, continues to be adequate for the job at hand; cf. Lemma 3.4. From this point on the proof proceeds as in [MT1].

\section{SharP $L^{p}$ RESULTS}

We continue to enforce the usual set of hypotheses on the manifold $M$, the metric tensor $g$, the domain $\Omega$, and the potential $V$. The goal is to further refine some of the results in $\S 4$ by developing an optimal $L^{p}$ theory for the Dirichlet and Neumann problems. Our main result in this regard is the following.

Theorem 5.1. There exists $\varepsilon=\varepsilon(\Omega, L)>0$ such that the boundary value problems

$$
u \in C_{l o c}^{1, \sigma}(\Omega), L u=0, \quad(\nabla u)^{*} \in L^{p}(\partial \Omega),\left.u\right|_{\partial \Omega}=f \in H^{1, p}(\partial \Omega),
$$

and

$$
\begin{gathered}
u \in C_{l o c}^{1, \sigma}(\Omega), \quad L u=0, \quad(\nabla u)^{*} \in L^{p}(\partial \Omega),\left.\partial_{\nu} u\right|_{\partial \Omega}=f \in L^{p}(\partial \Omega), \\
\int_{\Omega} u d V o l=0, \quad \int_{\partial \Omega} f d \sigma=0
\end{gathered}
$$

are well-posed for $1<p<2+\varepsilon$, whereas

$$
u \in C_{l o c}^{1, \sigma}(\Omega), L u=0, \quad u^{*} \in L^{q}(\partial \Omega),\left.\quad u\right|_{\partial \Omega}=f \in L^{q}(\partial \Omega)
$$

is well-posed for $2-\varepsilon<q<\infty$.

Uniqueness is seen much as in [MT2, while existence and estimates follow from Theorems 4.1 and 4.2, interpolation, and the proposition below.

Proposition 5.2. Assume that $u \in C_{l o c}^{1, \sigma}(\Omega)$ with $(\nabla u)^{*} \in L^{2}(\partial \Omega)$ satisfies $L u=0$ in $\Omega$, and either

$$
\operatorname{supp}\left(\left.\partial_{\nu} u\right|_{\partial \Omega}\right) \subset B_{r}\left(x_{0}\right) \cap \partial \Omega, x_{0} \in \partial \Omega, r>0, \quad\left\|\partial_{\nu} u\right\|_{L^{\infty}(\partial \Omega)} \leq r^{-(n-1)},
$$




$$
\operatorname{supp}\left(\left.u\right|_{\partial \Omega}\right) \subset B_{r}\left(x_{0}\right) \cap \partial \Omega, x_{0} \in \partial \Omega, r>0, \quad\left\|\nabla_{\tan } u\right\|_{L^{\infty}(\partial \Omega)} \leq r^{-(n-1)} .
$$

Then

$$
\left\|u^{*}\right\|_{L^{1}(\partial \Omega)}+\left\|(\nabla u)^{*}\right\|_{L^{1}(\partial \Omega)} \leq C,
$$

where $C=C(\partial \Omega)>0$ is a finite constant, independent of $u, x_{0}$, and $r$.

Similar results have been proved in [MT2, [MT4] (which further build on the work in $\mathrm{DK}], \mathrm{KP}]$ ), for smoother metric tensors (Lipschitz and Hölder continuous, respectively). The argument given there when (5.4) is satisfied carries over without any substantial alterations to the present setting, as the main ingredients (i.e., estimates on the Neumann kernel based on the De Giorgi-Nash-Moser theory, dyadic decompositions, the $L^{2}$ results of $\S 4$, and Caccioppoli's inequality) continue to work when the assumptions on the metric tensor are relaxed to the current hypotheses.

When (5.5) holds, much as in [MT2], MT4, we rely on a maximum principle argument. As in the works just mentioned, the key ingredient in establishing (5.6) is the construction of some suitable barrier functions, described in the following lemma.

Lemma 5.3. There exist $\gamma \in(0,1), C \in(0, \infty)$ and, for each $p \in \partial \Omega$, a function $\varphi_{p} \in C^{0}(\bar{\Omega} \backslash\{p\})$ such that

$$
\operatorname{dist}(x, p)^{2-n-\gamma} \leq \varphi_{p}(x) \leq C \operatorname{dist}(x, p)^{2-n-\gamma} \text { on } \bar{\Omega},
$$

and

$$
(\Delta-V) \varphi_{p} \leq 0 \quad \text { in } \Omega .
$$

In the remaining part of this section, we present the main steps in the construction of the functions $\varphi_{p}$. The initial part of the construction in the present case is the same as in MT2. For the convenience of the reader we recall how this is carried out. Starting with $p \in \partial \Omega$ arbitrary, choose a (large) neighborhood $U$ of $p$ and a coordinate system on $U$ centered at $p \equiv 0$ such that $g^{j k}(p)=\delta^{j k}$. Next, let $\Gamma_{p}$ be a truncated circular cone in $\mathbb{R}^{n} \backslash \Omega$, with vertex at the origin, continued to the infinite cone $C_{p}$. Working in polar coordinates on the complementary cone $K_{p}=\mathbb{R}^{n} \backslash C_{p}$, a cone over $\mathcal{O}_{p} \subset S^{n-1}$, we introduce

$$
\psi_{p}(r \omega):=r^{2-n-\gamma} \beta_{p}(\omega),
$$

where, with $\Delta_{S}$ the Laplace operator on $S^{n-1}$,

$$
\Delta_{S} \beta_{p}(\omega)+\gamma(n-2+\gamma) \beta_{p}(\omega)=-a_{1} \text { in } \mathcal{O}_{p},\left.\quad \beta_{p}\right|_{\partial \mathcal{O}_{p}}=b_{1}
$$

Then, if $b_{1}>0$ is large enough and $\gamma>0, a_{1}>0$ are sufficiently small, we have

$$
|x-p|^{2-n-\gamma} \leq \psi_{p}(x) \leq A|x-p|^{2-n-\gamma} \text { in } K_{p}
$$

and

$$
\Delta_{0} \psi_{p}(x)=-a_{1}|x-p|^{-n-\gamma} \text { in } K_{p},
$$

where $\Delta_{0}$ is the ordinary Euclidean Laplacian. Compare (6.23)-(6.29) of [MT2].

Now, if the metric tensor $g$ on $M$ is smooth enough, then $\Delta$ is a sufficiently gentle perturbation of $\Delta_{0}$ and the passage from $\psi_{p}$ to $\varphi_{p}$ is a simple affair; see the 
final arguments in the proof of Proposition 6.2 of [MT2]. In the present setting, the endgame requires further effort, and we now turn to this task.

For starters, let $\widetilde{\mathcal{O}}_{p} \subset S^{n-1}$ be a circular cap whose closure is contained in the interior of $\mathcal{O}_{p}$, and whose complement in $S^{n-1}$ still generates a cone whose intersection with $U$ meets $\bar{\Omega}$ only at $p$. Next, fix $\tilde{\beta}_{p} \in C_{0}^{\infty}\left(\mathcal{O}_{p}\right)$ which is equal to 1 on $\widetilde{\mathcal{O}}_{p}$, and pick $\alpha \in C_{0}^{\infty}(\mathbb{R}), \alpha(r)=1$ for $|r| \leq r_{0}$, and $\alpha(r)=0$ for $|r| \geq 2 r_{0}$, where $r_{0}$ is bounded away from zero but chosen so that $B_{3 r_{0}}(p) \subset U$. Then set

$$
\tilde{\psi}_{p}(r \omega)=\alpha(r) \tilde{\beta}_{p}(\omega) \psi_{p}(r \omega),
$$

so that $\tilde{\psi}_{p}$ can be regarded as a function on $M$ with support in $U$. Note that

$$
\left|\Delta_{0} \tilde{\psi}_{p}(x)\right| \leq C|x-p|^{-n-\gamma}, \quad\left|\nabla \tilde{\psi}_{p}(x)\right| \leq C|x-p|^{-n-\gamma+1},
$$

and

$$
\Delta_{0} \tilde{\psi}_{p}(x) \leq-a_{2}|x-p|^{-n-\gamma}+b_{2} \text { on } \Omega \cap U,
$$

since $\tilde{\psi}_{p}(r \omega)=\alpha(r) \psi_{p}(r \omega)$ on $\Omega \cap U$. Also, clearly,

$$
|x-p|^{2-n-\gamma} \leq \tilde{\psi}_{p}(x) \leq A|x-p|^{2-n-\gamma} \text { in } \Omega \cap B_{r_{0}}(p) .
$$

Now consider

$$
\Delta \tilde{\psi}_{p}-\Delta_{0} \tilde{\psi}_{p}=\partial_{j}\left(\left(g^{j k} g^{1 / 2}-\delta^{j k}\right) \partial_{k} \tilde{\psi}_{p}\right)=\partial_{j} \Lambda_{p}^{j}=\Psi_{p},
$$

where the last two equalities define $\Lambda_{p}^{j}$ and $\Psi_{p}$. Then $\Psi_{p}=\partial_{j} \Lambda_{p}^{j}$ is a distribution on $M$ with support in $U$, and

$$
\left|\Lambda_{p}^{j}(x)\right| \leq C \frac{\omega(|x-p|)}{|x-p|^{n-1+\gamma}} .
$$

In particular, $\Lambda_{p}^{j} \in L^{q}$ whenever $1<q<n /(n-1+\gamma)$. At this stage, we invoke Proposition 2.1 and solve

$$
L w_{p}=\Psi_{p} \in H^{-1, q}(M), \quad w_{p} \in H^{1, q}(M), \quad 1<q<\frac{n}{n-1+\gamma} .
$$

Note that, much as for (2.46), estimates for $w_{p}$ will follow from (2.57) with $s=$ $n-1+\gamma$. Thus, an analysis of $w_{p}$ parallel to $(2.46)-(2.59)$ yields the estimate

$$
\left|w_{p}(x)\right| \leq C \frac{\omega(|x-p|)}{|x-p|^{n-2+\gamma}} .
$$

Consequently,

$$
\left|w_{p}(x)\right| \leq C \omega(\operatorname{dist}(x, p)) \operatorname{dist}(x, p)^{-n+2-\gamma} .
$$

Hence, for an appropriate constant $B_{1}>0$,

$$
\varphi_{p}^{\#}(x)=\tilde{\psi}_{p}(x)-w_{p}(x)+B_{1}
$$

satisfies

$$
A_{1} \operatorname{dist}(x, p)^{2-n-\gamma} \leq \varphi_{p}^{\#}(x) \leq A_{2} \operatorname{dist}(x, p)^{2-n-\gamma} \text { on } \bar{\Omega},
$$

for some $A_{2}>A_{1}>0$, while

$$
L \varphi_{p}^{\#}=\Delta_{0} \tilde{\psi}_{p}-V \varphi_{p}^{\#}
$$

Thus,

$$
L \varphi_{p}^{\#} \leq-a_{3} \operatorname{dist}(x, p)^{-n-\gamma}+b_{3} \text { on } \Omega .
$$


Finally, to go from $\varphi_{p}^{\#}$ to the function $\varphi_{p}$ with the stated properties in Lemma 5.3 is routine. This finishes the proof of Proposition 5.2; as a corollary, at this point we have Theorem 5.1 .

Corollary 5.4. With the hypotheses of Theorem 5.1 enforced, there exists $\varepsilon=$ $\varepsilon(\partial \Omega)>0$ so that for $2-\varepsilon<p<\infty, 1 / p+1 / q=1$, the operators

$$
\frac{1}{2} I+K: L^{p}(\partial \Omega) \longrightarrow L^{p}(\partial \Omega), \quad \frac{1}{2} I+K: H^{1, q}(\partial \Omega) \longrightarrow H^{1, q}(\partial \Omega),
$$

and

$$
S: L^{q}(\partial \Omega) \longrightarrow H^{1, q}(\partial \Omega)
$$

are isomorphisms.

In particular, the solutions of the boundary value problems (5.1), (5.2) and (5.3) have natural integral representation formulas (cf. the discussion in §4).

Proof. The main ingredients are already in place; so we will only outline the main steps. First, there exists $\varepsilon>0$ so that, for each $1<q<2+\varepsilon$, the following Rellich type estimate holds:

$$
\left\|\nabla_{\tan } u\right\|_{L^{q}(\partial \Omega)} \approx\left\|\partial_{\nu} u\right\|_{L^{q}(\partial \Omega)}+\text { residual terms, }
$$

uniformly for $u$ such that $(\nabla u)^{*} \in L^{q}(\partial \Omega)$ and $L u=0$. Indeed, this is a consequence of the well-posedness of the Dirichlet problem with boundary data in $H^{1, q}(\partial \Omega)$ and the $L^{q}$-Neumann problem for $L$ in $\Omega$; cf. Theorem 5.1. In turn, when utilized both in $\Omega$ and in $M \backslash \bar{\Omega}$, this estimate and the jump-relations (3.9), (3.10), (3.12) lead to the conclusion that the operators under discussion are bounded from below, eventually modulo compact operators (cf., e.g., $\$ 7$ in [MT2 for details in similar circumstances). In particular, they are semi-Fredholm and, by invoking the $L^{p_{-}}$ theory with $p$ near 2 from $\S 4$, it follows that these operators are either onto, or injective with closed range. At this stage, Theorem 2.10 from [KM] yields the desired conclusion (much as in Theorem 7.1 and Theorem 7.3 of [MT2]).

Corollary 5.5. Retain the same hypotheses as in Theorem 5.1. Then there exists $\varepsilon=\varepsilon(\partial \Omega)>0$ so that the claims made in Theorem 4.2 are valid for $1<p<2+\varepsilon$.

Proof. This is a direct consequence of Corollary 5.4 and the proof of Theorem 4.2 .

\section{REFERENCES}

[Ca] A.P. Calderón, Cauchy integrals on Lipschitz curves and related operators, Proc. Nat. Acad. Sci. (USA) 74 (1977), 1324-1327. MR 57:6445

[CMM] R. Coifman, A. McIntosh, and Y. Meyer, L'intégrale de Cauchy definit un opérateur borné sur $L^{2}$ pour les courbes lipschitziennes, Ann. Math. 116 (1982), 361-388. MR 84m:42027

[DK] B. Dahlberg and C. Kenig, Hardy spaces and the Neumann problem in L $L^{p}$ for Laplace's equation in Lipschitz domains, Ann. of Math. 125 (1987), 437-465. MR 88d:35044

[DiF] G. DiFazio, $L^{p}$ estimates for divergence form elliptic equations with discontinuous coefficients, Boll. U.M.I. 10-A (1996), 409-420. MR 97e:35034

[Ed] R. E. Edwards, Functional Analysis. Theory and Applications, Corrected reprint of the 1965 original. Dover Publications, Inc., New York, 1995. MR 95k:46001

[FJR] E. Fabes, M. Jodeit, and N. Riviere, Potential techniques for boundary value problems in $C^{1}$ domains, Acta Math. 114 (1978), 165-186. MR 80b:31006

[GW] M. Grüter and K.-O. Widman, The Green function for uniformly elliptic equations, Manuscr. Math. 37 (1982), 303-342. MR 83h:35033 
[JK] D. Jerison and C. Kenig, Boundary value problems in Lipschitz domains, pp. 1-68 in "Studies in Partial Differential Equations," W. Littman, ed., MAA, 1982. MR 85f:35057

[KM] N. Kalton and M. Mitrea, Stability results on interpolation scales of quasi-Banach spaces and applications, Trans. Amer. Math. Soc. 350 (1998), 3903-3922. MR 98m:46094

[KP] C. Kenig and J. Pipher, The Neumann problem for elliptic equations with non-smooth coefficients, Invent. Math. 113 (1993), 447-509. MR 95b:35046

[Me] N. G. Meyers, An $L^{p}$-estimate for the gradient of solutions of second order elliptic divergence equations, Annali della Scuola Norm. Sup. Pisa, III, 17 (1963), 189-206. MR 28:2328

[MT1] M. Mitrea and M. Taylor, Boundary layer methods for Lipschitz domains in Riemannian manifolds, J. Funct. Anal. 163 (1999), 181-251. MR 2000b:35050

[MT2] M. Mitrea and M. Taylor, Potential theory on Lipschitz domains in Riemannian manifolds: $L^{p}$, Hardy, and Hölder space results, Communications in Analysis and Geometry, 9 (2001), 369-421. MR 2002f:31012

[MT3] M. Mitrea and M. Taylor, Potential theory on Lipschitz domains in Riemannian manifolds: Sobolev-Besov space results and the Poisson problem, J. Funct. Anal. 176 (2000), 1-79. MR 2002e:58044

[MT4] M. Mitrea and M. Taylor, Potential theory on Lipschitz domains in Riemannian manifolds: Hölder continuous metric tensors, Comm. PDE 25 (2000), 1487-1536. MR 2001h:35040

[P] J. Peetre, On convolution operators leaving $L^{p \lambda}$-spaces invariant, Ann. Mat. Pura Appl. 72 (1966), 295-304. MR 35:812

[Sp] S. Spanne, Some function spaces defined using the mean oscillation over cubes, Ann. Scuola Norm. Sup. Pisa 19 (1965), 593-608. MR 32:8140

[T1] M. Taylor, Pseudodifferential Operators and Nonlinear PDE, Birkhäuser, Boston, 1991. MR 92j:35193

[T2] M. Taylor, Microlocal analysis on Morrey spaces, in Singularities and Oscillations (J. Rauch and M. Taylor, eds.), IMA Vol. 91, Springer-Verlag, New York, 1997, pp. 97135. MR 99a:35010

[T3] M. Taylor, Tools for PDE: Pseudodifferential Operators, Paradifferential Operators, and Layer Potentials, Mathematical Surveys and Monographs, 81, Amer. Math. Soc., Providence, RI, 2000. MR 2001g:35004

[Ve] G. Verchota, Layer potentials and regularity for the Dirichlet problem for Laplace's equation in Lipschitz domains, J. Funct. Anal. 59 (1984), 572-611. MR 86e:35038

Department of Mathematics, University of Missouri, Columbia, Missouri 65211

E-mail address: marius@math.missouri.edu

Department of Mathematics, University of North Carolina, Chapel Hill, North CarOLINA 27599

E-mail address: met@math.unc.edu 